\section{(2) OPEN ACCESS}

- Supplemental material is published online only. To view, please visit the journal online (http://dx.doi.org/10.1136/ gutjnl-2020-320980).

For numbered affiliations see end of article.

\section{Correspondence to}

Pradeep Bhandari

Gastroenterology, Queen

Alexandra Hospital, Portsmouth P06 3LY, UK;

pradeep.bhandari@porthosp. nhs.uk

Received 24 February 2020 Revised 19 September 2020 Accepted 12 October 2020 Published Online First 19 November 2020
Check for updates

(c) Author(s) (or their employer(s)) 2021. Re-use permitted under CC BY-NC. No commercial re-use. See rights and permissions. Published by BMJ.

To cite: Kandiah $\mathrm{K}$,

Subramaniam $\mathrm{S}$,

Thayalasekaran S, et al. Gut 2021:70:1685-1691.

\title{
Multicentre randomised controlled trial on virtual chromoendoscopy in the detection of neoplasia during colitis surveillance high-definition colonoscopy (the VIRTUOSO trial)
}

\author{
Kesavan Kandiah (0) ${ }^{1,2}$ Sharmila Subramaniam, ${ }^{1}$ Sreedhari Thayalasekaran, ${ }^{1}$ \\ Fergus JQ Chedgy, ${ }^{1,3}$ Gaius Longcroft-Wheaton (D) , ${ }^{1}$ Carole Fogg (D) , \\ James F Brown, ${ }^{4}$ Samuel CL Smith (D, ${ }^{5}$ Marietta lacucci, ${ }^{5,6}$ Pradeep Bhandari ${ }^{1}$
}

\section{ABSTRACT}

Background Longstanding colonic IBD increases the risk of developing colorectal cancer. The utility of chromoendoscopy with standard-definition white light technology has been established. However, the use of high-definition virtual chromoendoscopy (HDV) in colitis surveillance remains undefined.

Objective To compare the performance of HDV (i-scan OE mode 2) with high-definition white light (HDWL) for detection of neoplasia in patients with IBD undergoing surveillance colonoscopy. Additionally, we assessed the utility of protocol-guided quadrantic non-targeted biopsies. Design A multioperator randomised controlled trial was carried out in two centres in the UK. Total of 188 patients (101 men, mean age 54) with longstanding ulcerative or Crohn's colitis were randomised, prior to starting the surveillance colonoscopy, to using either HDV ( $n=94)$ or HDWL ( $n=94)$ on withdrawal. Targeted and quadrantic nontargeted biopsies were taken in both arms per-randomisation protocol. The primary outcome was the difference in neoplasia detection rate (NDR) between HDV and HDWL. Results There was no significant difference between HDWL and HDV for neoplasia detection. The NDR was not significantly different for HDWL (24.2\%) and HDV $(14.9 \%)(p=0.14)$. All intraepithelial neoplasia (IEN) detected contained low-grade dysplasia only. A total of 6751 non-targeted biopsies detected one IEN only. The withdrawal time was similar in both arms of the study: median of $24 \mathrm{~min}$ (HDWL) versus $25.5 \mathrm{~min}$ (HDV). Conclusion HDV and HDWL did not differ significantly in the detection of neoplasia. Almost all neoplasia were detected on targeted biopsy or resection. Quadrantic non-targeted biopsies have negligible additional gain. Trial registration number Clinical Trial.gov ID NCT02822352.

\section{INTRODUCTION}

Longstanding colonic IBD increases the risk of developing colorectal cancer (CRC). The estimated prevalence of CRC in patients with longstanding UC is $3.7 \%$ (95\% CI $3.2 \%$ to $4.2 \%) .{ }^{1}$ The risk of developing CRC is similarly high in patients with longstanding Crohn's colitis (CC). ${ }^{2}$ The duration of the disease is directly proportional to the rates
Significance of this study

What is already known on this subject?

- Longstanding ulcerative and Crohn's colitis increases the risk of developing colorectal cancer (CRC).

- Surveillance colonoscopy is recommended by national and international guidelines to reduce the risk of developing CRC.

- Chromoendoscopy using standard-definition endoscopes has been shown to improve neoplasia detection rate, but the uptake of this technique has been low.

- The role of high-definition white light (HDWL) endoscopy and a novel high-definition virtual chromoendoscopy (i-scan OE Mode 2) is not known.

What are the new findings?

- Our randomised controlled trials did not demonstrate the superiority of high-definition virtual chromoendoscopy (HDV) over HDWL.

- The control group (HDWL) outperformed the intervention group (HDV) where targeted biopsies with HDWL alone can detect all the neoplasia in colitic bowel.

- The yield of quadrantic non-targeted biopsies in detecting neoplasia is extremely low. A total of 6751 non-targeted colonic biopsies detected one neoplasia in this study.

How might it impact on clinical practice in the foreseeable future?

- Targeted biopsies with HDWL endoscopy systems may be adequate in detecting neoplasia in the colitis surveillance population.

of developing CRC, with a variable cumulative incidence of $1 \%-2 \%$ by 10 years, $4 \%-8 \%$ by 20 years and $14 \%-18 \%$ by 30 years. ${ }^{3}$ Therefore, national and international guidelines recommend patients with colonic IBD of $8-10$ years duration to be enrolled in an endoscopic surveillance programme. $^{45}$ 
These guidelines recommend surveillance colonoscopy using chromoendoscopy with targeted biopsy as standard practice. Several studies have demonstrated up to threefold increase in neoplasia detection (per-patient basis) using chromoendoscopy when compared with standard-definition white light endoscopy. ${ }^{6-9}$ Despite the higher neoplasia yield by chromoendoscopy, the technique has not been widely adopted by endoscopists. This is partly due to the perceived additional time taken to carry out chromoendoscopy, lack of validated standards and training on the chromoendoscopy technique and inability to use the dyes in the presence of poor bowel preparation. ${ }^{10}$

The seminal trials showing the efficacy of chromoendoscopy in the detection of neoplasia in colitis were conducted using standard-definition white light technology. Carrying out surveillance colonoscopy using high-definition white light (HDWL) has been demonstrated to have a higher neoplasia detection rate (NDR) when compared with standard-definition white light. ${ }^{11}$ The per-patient NDR using HDWL in two recent trials was between 0.12 and 0.26 , an improvement from the per-patient NDR of 0.07 with chromoendoscopy using standard-definition white light in the older studies. ${ }^{612-14}$

Advances in technology have led to the development of, dyefree virtual chromoendoscopy technologies such as HD i-scan (Pentax, Tokyo, Japan) and NBI (Olympus, Tokyo, Japan). These technologies enhance the mucosal surface details, thereby theoretically improving the detection of neoplasia. However, two randomised controlled trials (RCTs) assessing the effectiveness of virtual chromoendoscopy over dye spray chromoendoscopy have been inconclusive. ${ }^{13} 15$

The i-scan OE-mode 2 (Pentax, Tokyo, Japan) is a highdefinition virtual chromoendoscopy (HDV) system that uses a combination of bandwidth-limiting filter and digital postprocessing technology. This technology uses red light emission as well as emission at $415 \mathrm{~nm}$ and $540 \mathrm{~nm}$ to enhance the surface of mucosa and the underlying blood vessels while maintaining a bright overall appearance, thus making it potentially efficient in detecting mucosal lesions in the colon.

The aim of this study was to evaluate the performance of HDV when compared with HDWL in patients with longstanding colonic IBD undergoing surveillance colonoscopy for neoplasia and to evaluate the additional yield of neoplasia detection with protocol-guided quadrantic but non-targeted colonic biopsies.

\section{METHODS}

Study design

This was a multicentre prospective RCT. The study participants were recruited from two centres in the UK (Queen Alexandra Hospital, Portsmouth and University Hospital Birmingham, Birmingham) between August 2016 and February 2018 (see online supplemental file 1)—study protocol).

\section{Patient and public involvement}

Patients and member of public were involved in the trial development stage. The group is representative of the local population eligible for the trial, and included patients or carers with bowel disease. We discussed the trial questions, acceptability of trial designs including parallel group versus cross-over and methods of recruitment. This group also reviewed patient-facing documents such as the informed consent and information sheet.

\section{Patients}

All consecutive patients with IBD above the age of 18 years with longstanding colitis (UC: 8 years after onset of symptoms for patients with subtotal or total colitis and 10 years after onset of symptoms for patients with left-sided colitis, CC: 8 years after diagnosis) referred for a surveillance colonoscopy and who were able to provide consent were invited to participate.

Exclusion criteria were persistent coagulopathy, known colonic neoplasia, pregnant women, fulminant colitis and inability to consent. Persistent coagulopathy was defined as patients who were on warfarin and had an international normalised ratio (INR) of $>1.5$ despite cessation of the drug or had not stopped their direct oral anticoagulants as per the British Society of Gastroenterology and European Society of Gastrointestinal Endoscopy guidelines on endoscopy in patients on anticoagulant therapy. ${ }^{16}$

\section{Procedure}

All patients received standard polyethylene glycol-based bowel preparation prior to the procedure. The patients were consented for the study following consent for the surveillance colonoscopy. Thereafter, demographic and clinical data were obtained. Patients were randomised on a 1:1 ratio to two study arms via an online randomisation platform (Sealed Envelope, UK) using random permuted blocks of randomly varying sizes prior to commencing the surveillance colonoscopy. The disease activity and bowel preparation were assessed during the insertion phase of the surveillance colonoscopy. Patients with active colitis (defined as modified Baron's score of $<3$ and Simple Endoscopic Score for Crohn's Disease of $<6$ ) and poor bowel preparation (defined as $>20 \%$ of the mucosa obscured by solid stool after suction) were withdrawn from the study.

All colonoscopies were carried out using i10 series Pentax colonoscopes without zoom and OPTIVISTA EPK-i7010 highdefinition video processors (Pentax, Germany), which were connected to high-definition monitors. The colonoscopies were undertaken by six dedicated endoscopists who have been trained to perform IBD surveillance colonoscopy by the two senior endoscopists in the study (PB and $\mathrm{MI}$ ). All the endoscopists involved had carried out more than a 100 cases using HDV and HDWL prior to the commencement of the study. The procedures were performed with the patients under conscious sedation using intravenous midazolam and fentanyl.

Intubation up to the caecum was carried out using HDWL in both arms. Once the caecum was reached, withdrawal was carried out using i-scan OE mode 2 for patients randomised to the HDV arm. For patients in the HDWL arm, withdrawal was carried out using HDWL only. A research nurse who was present for the whole duration of the procedure documented the times for the start of the procedure, caecal intubation and the end of the procedure where the colonoscope has been completely withdrawn from the patient using an electronic clock.

\section{Biopsy protocol}

The assessment for lesions was carried out on withdrawal of the endoscope from the caecum. The size, location and Paris classification of all lesions detected in both arms of the study were documented. These lesions were either resected or targeted biopsies were taken. No additional imaging technologies or techniques were used to assess the lesions prior to resection or biopsy. In addition, quadrantic non-targeted biopsies were taken every $10 \mathrm{~cm}$ in all patients in both arms of the study, starting at the caecum.

\section{Histopathological assessment}

Pathological specimens were analysed by the pathologist of each centre for the presence of neoplasia. A second pathologist expert in gastrointestinal pathology reviewed all specimens containing neoplasia. The following pathological diagnoses were considered 


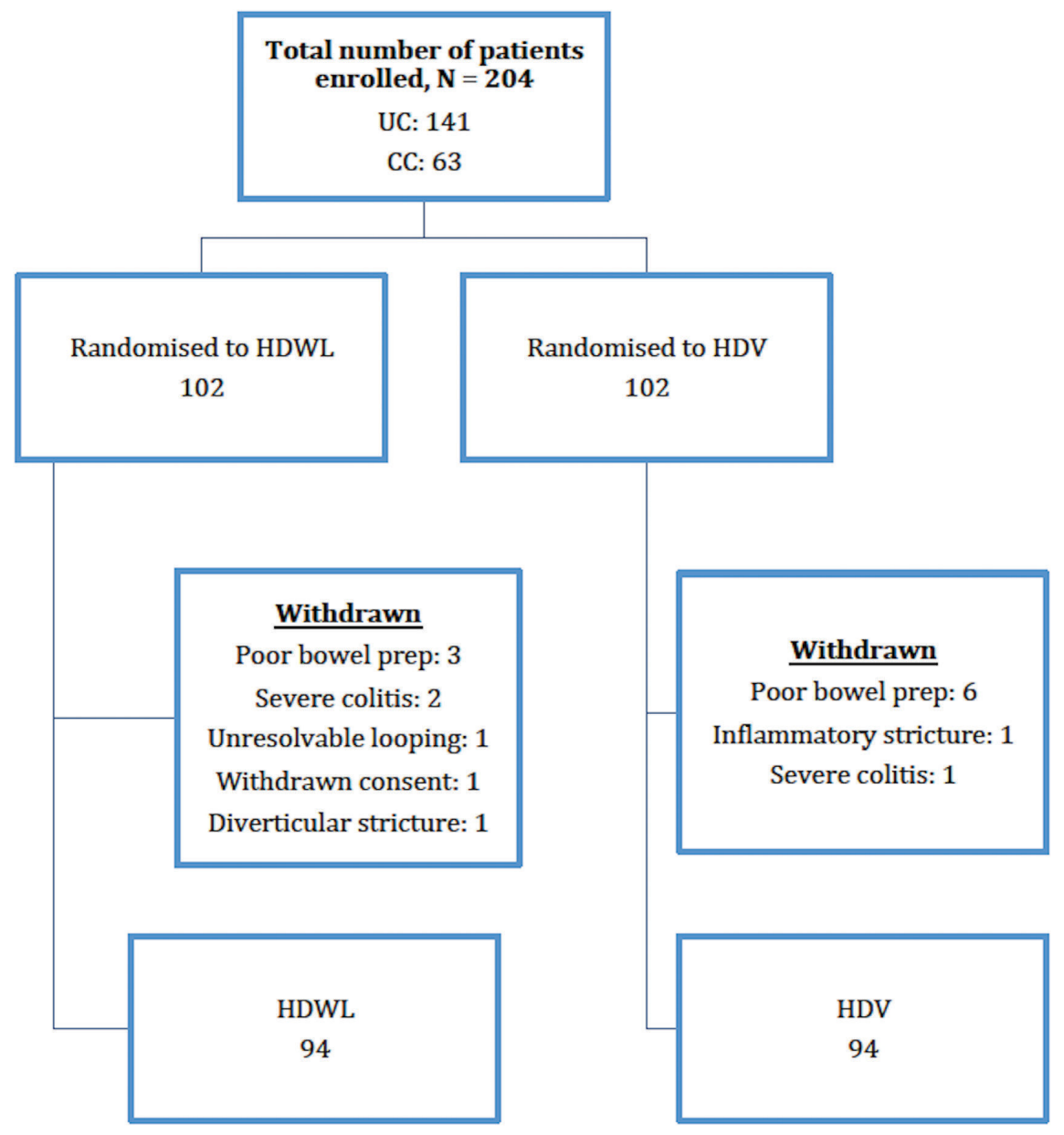

Figure 1 Study flowchart. CC, Crohn's colitis; HDV, high-definition virtual chromoendoscopy; HDWLE, high-definition white light.

to be neoplastic: adenoma, any grade of dysplasia, serrated or adenocarcinoma. Hyperplastic, regenerative, inflammatory and pseudopolyps were considered as non-neoplastic.

\section{STATISTICAL ANALYSIS}

In previous studies using the older virtual chromoendoscopy technologies, the NDR using HDV has been reported to be between $12 \%$ and $39 \% .{ }^{17-19}$ We have used an expected NDR in the HDV arm of $24 \%$ and of $9 \%$ in the HDWL (control) arm. With a significance level (alpha) of $5 \%$ and power of $80 \%$, the number needed was 93 in each group, leading to a total of 186 . However, we anticipated a $10 \%$ attrition rate after the colonoscopy has been started (eg, poor bowel preparation, endoscopically confirmed moderate to severe colitis). To adjust for this, we decided to recruit 204 patients.

The rate of neoplastic lesions detected with either endoscopic technique was analysed using a statistics software programme (SPSS V.24.0, IBM Armonk, New York, USA). The analyses were conducted on a 'per-patient' and 'per-lesion' basis. The 'per-patient' analysis was done by calculating the percentage of patients in whom true neoplastic lesions were detected from biopsies of endoscopically suspicious lesions. On the other hand, in 'per-lesion' analysis, the percentage of neoplastic lesion among all endoscopically suspicious lesions was calculated. Fisher's exact test and $\chi^{2}$ test, where deemed appropriate, were applied for dichotomous variables. A MannWhitney U test or Student's t-test was used for continuous variables. $P$ values $<0.05$ were considered significant for the analysis.

\section{RESULTS}

We enrolled 204 patients in the study between August 2016 and February 2018. Sixteen patients were excluded after randomisation due to poor bowel preparation, severe colitis, impassable inflammatory stricture, unresolvable looping, impassable diverticular stricture and withdrawal of consent. (figure 1). A total of 184 patients (92 in the HDWL and 92 in the HDV arms) were included in the final analysis. The baseline characteristics of patients in both arms did not differ (table 1). There were no adverse events in this study.

\section{Primary endpoint}

A total of 27 neoplastic lesions were detected in 22 patients in the HDWL arm ( $\mathrm{n}=92)$ and 25 neoplastic lesions were detected in 14 patients in the HDV arm $(n=92)$. The NDR was slightly higher in the HDWL arm (23.4\%) when compared with the HDV arm $(14.9 \%)$ but the difference was not statistically significant $(p=0.14)$. (table 2) An example of a neoplastic lesion detected using HDWL and HDV is shown in figure 2. The number of patients in each arm with neoplastic lesions and the characteristics of the lesions detected are detailed in table 3 .

\section{Secondary endpoints}

The mean neoplasia per-patient in the HDWL arm was 0.29 and in the HDV arm was 0.26 . This difference was not statistically significant $(p=0.75)$. The number of non-targeted quadrantic biopsies taken in the HDWL and HDV arm was 3373 and 3378, 
Table 1 Baseline patient characteristics

\begin{tabular}{|c|c|c|c|}
\hline & $\begin{array}{l}\text { HDWL } \\
\text { OR }(95 \% \mathrm{Cl})\end{array}$ & $\begin{array}{l}\text { HDV } \\
\text { OR }(95 \% \mathrm{Cl})\end{array}$ & Fisher's exact $p$ value \\
\hline Age in years, median (IQR, p25-p75) & 53.7 (20 to 79$)$ & 54.3 (21 to 80$)$ & 0.786 \\
\hline Sex (M:F) & $46: 48$ & $55: 39$ & 0.242 \\
\hline \multicolumn{4}{|l|}{ Type of colitis, n (\%) } \\
\hline UC & $60(64)$ & $69(73)$ & 0.204 \\
\hline Concurrent primary sclerosing cholangitis & $1(1)$ & $1(1)$ & \\
\hline Crohn's colitis & $33(35)$ & $24(26)$ & 0.204 \\
\hline \multicolumn{4}{|l|}{ Distribution of colitis, $n(\%)$} \\
\hline Left sided & $15(16)$ & $23(24)$ & 0.203 \\
\hline Subtotal/total & $79(84)$ & $71(76)$ & 0.203 \\
\hline Duration of illness in years, median (IQR, p25-p75) & $17.0(13$ to 27.5$)$ & 18.0 (13.3 to 25.8$)$ & 0.635 \\
\hline Left sided & $19.0(15.5$ to 22.5$)$ & $23.0(17.5$ to 26.0$)$ & \\
\hline Subtotal/total & $17.0(12.5$ to 28.0$)$ & $16.0(12.5$ to 25.5$)$ & \\
\hline \multicolumn{4}{|l|}{ Therapy, n (\%) } \\
\hline Steroids & $2(2)$ & $1(1)$ & 1 \\
\hline ASA & $76(81)$ & $80(85)$ & 0.561 \\
\hline Immunosuppressant & $29(31)$ & $27(29)$ & 0.873 \\
\hline Biologics & $9(10)$ & $6(6)$ & 0.592 \\
\hline
\end{tabular}

ASA, aminosalicylic acid; HDV, high-definition virtual chromoendoscopy; HDWLE, high-definition white light.

respectively. The mean number of biopsies taken per-patient was 35.9 in each arm. Only one neoplasia (low-grade dysplasia within a segment of active colitis in the HDWL arm) was detected on quadrantic non-targeted biopsies in this study, otherwise all neoplasias were detected on targeted biopsy.

The number of sessile serrated lesions (SSL) detected was 13 in the HDWL arm and 4 in the HDV arm of the study. These lesions were all located in the right colon; nine in the transverse colon, seven in the ascending colon and one in the caecum. On a per-patient analysis, SSLs were seen more frequently in the HDWL arm (8/98 vs 4/98 patients).

There was no difference in the total withdrawal time in either arm of the study; 24.0 (IQR 20.0-30.0) $\mathrm{min}$ in the HDWL arm and 25.5 (IQR 21.0-31.0) $\mathrm{min}$ in the HDV arm $(\mathrm{p}=0.216)$. The difference in the mean total withdrawal time in patients where targeted lesions were biopsied or resected in either study arm was not statistically significant; 29.0 (IQR 22.8-31.3) $\mathrm{min}$ in the HDWL arm and 31.6 (IQR 25.0-37.0) $\mathrm{min}$ in the HDV arm $(\mathrm{p}=0.11)$.

\section{DISCUSSION}

In this multioperator multicentre randomised control trial, we did not demonstrate a significant difference between HDV and HDWL in the detection of neoplasia in patients with longstanding IBD. Furthermore, we did not find any difference in the total number of neoplasia and the ratio of non-neoplastic to neoplastic lesions detected in either arm of the study. To our knowledge, this is the first multicentre randomised control trial

\begin{tabular}{|c|c|c|}
\hline & $\begin{array}{l}\text { HDWL } \\
(n=94)\end{array}$ & $\begin{array}{l}\text { HDV } \\
(n=94)\end{array}$ \\
\hline Total number of targeted lesions detected & 71 & 78 \\
\hline Number of neoplastic lesions detected & 27 & 25 \\
\hline Number of patients with at least one neoplastic lesion & 22 & 14 \\
\hline Neoplasia detection rate & $23.4 \%$ & $14.9 \%$ \\
\hline
\end{tabular}

Neoplasia detection rate: Fisher's exact $\mathrm{p}$ value $=0.14$.

HDV, high-definition virtual chromoendoscopy; HDWLE, high-definition white light. assessing the utility of a novel i-scan OE mode 2 in the detection of neoplasia during colitis surveillance.

The NDR in this study was similar to other studies investigating the utility of NBI. ${ }^{1520}$ However, unlike these studies, a larger proportion of the neoplastic lesions detected in our study were flat (Paris IIa/IIb): $81 \%$ versus $32 \%-58 \%$ in previously reported literature. This is possibly due to the overall bright appearance of the mucosa in both arms of this study that is not usually seen easily with narrow band technologies due to the darkening effects of these technologies. In addition, more intraepithelial neoplasias were detected with HDV and HDWL detected more SSLs. SSLs tend to have mucous caps, thereby obscuring the surface patterns altogether. With HDV, faecal residue and mucous have the same colour. The small numbers of these lesions make it difficult to make meaningful statistical conclusions.

This study did not compare chromoendoscopy versus HDWL. However, previous guidelines including Surveillance for Colorectal Endoscopic Neoplasia Detection and Management in Inflammatory Bowel Disease Patients: International Consensus Recommendations (SCENIC) recommend the use of chromoendoscopy based on the evidence from a single observational study that found that the NDR with HDWL alone was 9\% and when used with chromoendoscopy, the NDR was $21 \% .^{21} 22$ The findings in our RCT show that HDWL on its own has an NDR of $23.4 \%$. This detection rate is comparable to other established techniques in colitis surveillance colonoscopy including high-definition chromoendoscopy. ${ }^{1415202324}$ This will certainly add to the discussion in future guidelines that HDWL on its own may be sufficient to carry out an effective colitis surveillance colonoscopy.

International IBD surveillance guidelines recommend chromoendoscopy in IBD surveillance. However, adherence to these guidelines has been poor. ${ }^{2526}$ The basic minimum for IBD surveillance colonoscopy has been and still is quadrantic nontargeted colonic biopsies. In a recent survey among international IBD physicians and endoscopists, up to $43 \%$ of respondents stated that they perform non-targeted colonic biopsies in $>75 \%$ of cases when performing surveillance with chromoendoscopy. ${ }^{10}$ In our study, a total of 6751 non-targeted biopsies were taken and only 1 was reported to contain neoplasia (low-grade 


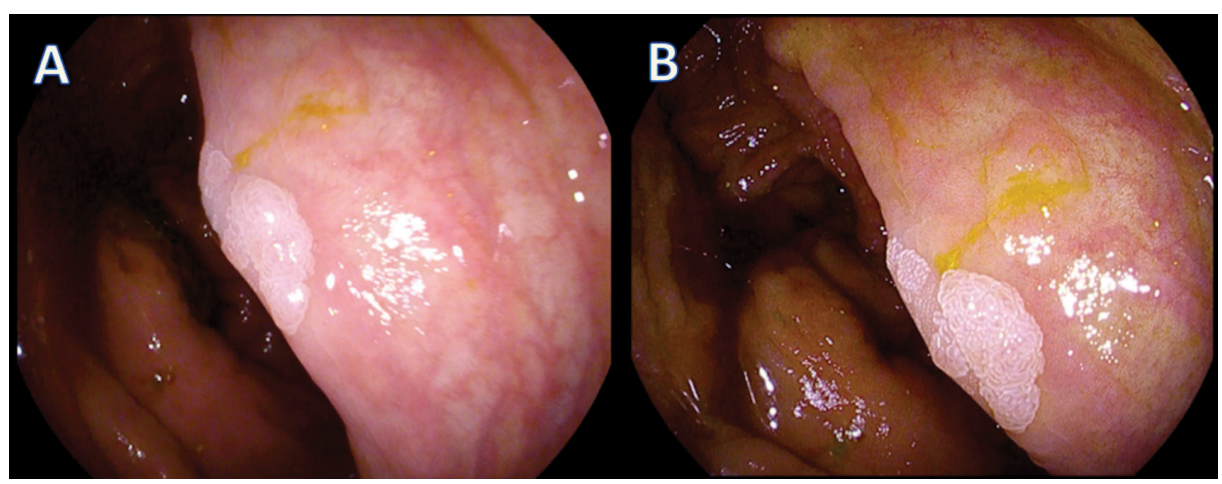

Figure 2 Example of a neoplastic lesion detected with HDWL and HDV. In picture (A), the lesion is seen using HDWL and in picture (B), the same lesion is seen using HDV. The histology of this lesion was tubular adenoma with low grade dysplasia. HDV, high-definition virtual chromoendoscopy; HDWLE, high-definition white light.

dysplasia with active background disease). Our findings add to the body of evidence on the futility of non-targeted biopsies in detecting neoplasia. ${ }^{27}$ In the era of high-definition endoscopy systems, it is time to abandon costly and ineffective quadrantic non-targeted biopsies during IBD surveillance colonoscopy.

Increased withdrawal times have been shown to increase adenoma detection rates in colonoscopies done on patients with non-IBD. ${ }^{29}$ In our study, the withdrawal time was between a median of 24.0 and $25.5 \mathrm{~min}$ in each study arm. This is likely to be due to the 6751 non-targeted biopsies that were taken as part of the study protocol. The design of our study allows us to conclude that quadrantic non-targeted biopsies do not make any significant additional contribution to NDR over and above the targeted biopsies. However, we cannot completely exclude the impact of long withdrawal time on NDR rate in our study. If quadrantic non-targeted biopsies were to be abandoned, the withdrawal times could be shortened. A recent systematic review and experts' opinion recommends that the withdrawal time of $17 \mathrm{~min}$ should be considered as one of the key performance indexes of

Table 3 The number of patients with lesions and the characteristics of lesions detected

\begin{tabular}{|c|c|c|c|}
\hline & HDWL $(n=94)$ & HDV $(n=94)$ & $\begin{array}{l}\text { Fisher's exact } \\
\text { p value }\end{array}$ \\
\hline $\begin{array}{l}\text { Total number of lesions } \\
\text { identified }\end{array}$ & 71 & 78 & 0.21 \\
\hline $\begin{array}{l}\text { Total number of neoplastic } \\
\text { lesions }\end{array}$ & 27 & 25 & 0.49 \\
\hline \multicolumn{4}{|l|}{ Paris classification of lesions } \\
\hline Ila & 18 & 19 & 0.55 \\
\hline Ilb & 2 & 3 & 0.32 \\
\hline Ila-c & 1 & 0 & 0.33 \\
\hline Is & 6 & 3 & 0.33 \\
\hline $\begin{array}{l}\text { Size of neoplastic lesions } \\
\text { in } \mathrm{mm} \text {, median (range) }\end{array}$ & $4.30(2-15)$ & $3.16(1-20)$ & 0.24 \\
\hline \multicolumn{4}{|c|}{ Number of lesions according to histology } \\
\hline $\begin{array}{l}\text { Sessile serrated lesions } \\
\text { (without dysplasia) }\end{array}$ & 13 & 4 & \\
\hline \multicolumn{4}{|c|}{ Intraepithelial neoplasia (colitis areas) } \\
\hline Low grade & 13 & 22 & \\
\hline High grade & 0 & 0 & \\
\hline Adenocarcinoma & 1 & 0 & \\
\hline
\end{tabular}

colitis surveillance. ${ }^{30}$ Therefore, it may be beneficial for patients' undergoing colitis surveillance to be allocated extra time to allow for careful assessment of the colonic mucosa during withdrawal.

One of the strengths of this study is the randomised multicentre design involving several endoscopists. While previous studies were often carried out in tertiary referral centres, patients in this study were recruited through a general IBD surveillance population. An additional strength of our study is that quadrantic nontargeted biopsies were taken in both arms, thereby reducing the risk of missing 'invisible' neoplastic lesions.

There were several limitations to this study. As it is a parallel study, it was not possible to calculate the miss rate of neoplasia. However, a cross-over trial will require a long washout period and a second colonoscopy. A back-to-back trial would introduce a 'double-look' bias. Both of these trial designs were found to be unacceptable to our patient and public involvement group. The second limitation is that although the participating endoscopists were experienced in the use of advanced imaging technologies including i-scan and in IBD surveillance, there was no validated classification for characterisation of neoplastic lesions in IBD colon with i-scan at the time the study was conducted. Despite this, however, the NDR was comparable to other similar studies. The NDR in the HDV group was lower compared with other studies. However, non-targeted biopsies in the HDV group did not pick up any additional neoplasia. Our trial design that includes targeted and non-targeted quadrantic biopsies provide the confidence to state that the lower HDV detection rate was not due to high miss rate of HDV.

Patients recruited in this study were not stratified based on their type of IBD or by gender. Therefore, the imbalance of the number of patients with UC and the male preponderance of patients in the HDV arm of the study arose by chance. The study was focused on the overall detection by the different imaging platforms, and therefore, we did not focus on gender or specific lesions identified. There is no convincing evidence in the literature that such gender differences occur or that lesions are different in UC versus CC. An interesting finding was that the neoplasia per-patient detected was higher with HDV (25 lesions in 14 patients) than it was with HDWL (27 lesions in 22 patients). This is a useful marker of efficacy but it has not been adopted by other similar studies, thereby making it difficult to compare and contrast the study findings. Additionally, our study was not powered based on the neoplasia per-patient rate. A meaningful deduction, therefore, cannot be made based on this finding. 
In conclusion, in this multicentre RCT, we could not demonstrate a significant difference in NDR between HDV (i-scan OE mode 2) and HDWL. However, IBD surveillance using a high-definition endoscopy system achieved high NDR. This technology on its own may be sufficient to detect neoplasia in patients with longstanding IBD.

\section{Author affiliations}

'Department of Gastroenterology, Portsmouth Hospitals University NHS Trust, Portsmouth, UK

${ }^{2}$ Department of Gastroenterology, St George's University Hospitals NHS Foundation Trust, London, UK

${ }^{3}$ Digestive Diseases Centre, Brighton and Sussex University Hospitals, Brighton, UK ${ }^{4}$ School of Pharmacy and Biomedical Sciences, University of Portsmouth, Portsmouth, UK ${ }^{5}$ Institute of Immunology and Immunotherapy, NIHR Wellcome Trust Clinical Research Facilities, University of Birmingham, Birmingham, UK

${ }^{6}$ National Institute for Health Research (NIHR) Birmingham Biomedical Research Centre, University of Birmingham, Birmingham, UK

Twitter Carole Fogg @carole_fogg

Acknowledgements The authors would like to thank Ms Lisa Murray, Dr Michelle Baker-Moffatt, Ms Joanne Dash and Ms Hayley Downe in assisting with the recruitment of patients into the study. We would like to also thank Mr Paul Bassett for his assistance in the statistical analysis of the study.

Contributors KK participated in the study conception, patient recruitment, data collection, statistical analysis, writing of the manuscript and the final approval of the manuscript. SS participated in the patient recruitment, data collection, statistical analysis and critical analysis of the manuscript. CF, JFB, GL-W participated in the study concept, design and critical revision of the manuscript. FJQC, ST and SCLS participated in the patient recruitment and data collection. Ml participated in the patient recruitment, data collection, critical revision and final approval of the manuscript. PB participated in the study concept, study design, data analysis, critical revision and final approval of the manuscript.

Funding An unrestricted grant by Pentax UK Ltd was provided in the form of a loan of endoscopes and an endoscopy processor.

Competing interests None declared.

Patient consent for publication Not required.

Ethics approval Ethical approval for the study was obtained (REC reference number 16/SC/0116) and the study was adopted on to the National Institute of Health Research (NIHR) portfolio (UKCRN ID: 317157).

Provenance and peer review Not commissioned; externally peer reviewed.

\section{Data availability statement}

Deidentified participant data are available upon reasonable request. Request to be made in writing to the corresponding author.

Supplemental material This content has been supplied by the author(s). It has not been vetted by BMJ Publishing Group Limited (BMJ) and may not have been peer-reviewed. Any opinions or recommendations discussed are solely those of the author(s) and are not endorsed by BMJ. BMJ disclaims all liability and responsibility arising from any reliance placed on the content. Where the content includes any translated material, BMJ does not warrant the accuracy and reliability of the translations (including but not limited to local regulations, clinical guidelines, terminology, drug names and drug dosages), and is not responsible for any error and/or omissions arising from translation and adaptation or otherwise.

Open access This is an open access article distributed in accordance with the Creative Commons Attribution Non Commercial (CC BY-NC 4.0) license, which permits others to distribute, remix, adapt, build upon this work non-commercially, and license their derivative works on different terms, provided the original work is properly cited, appropriate credit is given, any changes made indicated, and the use is non-commercial. See: http://creativecommons.org/licenses/by-nc/4.0/.

\section{ORCID iDs}

Kesavan Kandiah http://orcid.org/0000-0001-9447-3172

Gaius Longcroft-Wheaton http://orcid.org/0000-0001-9899-9948

Carole Fogg http://orcid.org/0000-0002-3000-6185

Samuel CL Smith http://orcid.org/0000-0001-8351-1081

\section{REFERENCES}

1 Eaden JA, Abrams KR, Mayberry JF. The risk of colorectal cancer in ulcerative colitis: a meta-analysis. Gut 2001;48:526-35.

2 Gillen CD, Andrews HA, Prior P, et al. Crohn's disease and colorectal cancer. Gut 1994;35:651-5.

3 Herrinton LJ, Liu L, Levin TR, et al. Incidence and mortality of colorectal adenocarcinoma in persons with inflammatory bowel disease from 1998 to 2010. Gastroenterology 2012;143:382-9.
4 Cairns SR, Scholefield JH, Steele RJ, et al. Guidelines for colorectal cancer screening and surveillance in moderate and high risk groups (update from 2002). Gut 2010;59:666-89.

5 Maaser C, Sturm A, Vavricka SR, et al. ECCO-ESGAR guideline for diagnostic assessment in IBD Part 1: initial diagnosis, monitoring of known IBD, detection of complications. J Crohns Colitis 2019;13:144-64.

6 Rutter MD, Saunders BP, Schofield G, et al. Pancolonic indigo carmine dye spraying for the detection of dysplasia in ulcerative colitis. Gut 2004;53:256-60

7 Marion JF, Waye JD, Present DH, et al. Chromoendoscopy-targeted biopsies are superior to standard colonoscopic surveillance for detecting dysplasia in inflammatory bowel disease patients: a prospective endoscopic trial. Am J Gastroenterol 2008;103:2342-9.

8 Kiesslich R, Fritsch J, Holtmann M, et al. Methylene blue-aided chromoendoscopy for the detection of intraepithelial neoplasia and colon cancer in ulcerative colitis. Gastroenterology 2003;124:880-8.

9 Kiesslich R, Goetz M, Lammersdorf K, et al. Chromoscopy-guided endomicroscopy increases the diagnostic yield of intraepithelial neoplasia in ulcerative colitis. Gastroenterology 2007;132:874-82.

10 Kaltenbach TR, Soetikno RM, DeVivo R, et al. Optimizing the quality of endoscopy in inflammatory bowel disease: focus on surveillance and management of colorectal dysplasia using interactive image- and video-based teaching. Gastrointest Endosc 2017:86:1107-17

11 Subramanian V, Telakis E, Ramappa V, et al. Comparison of high definition with standard white light endoscopy for detection of dysplastic lesions during surveillance colonoscopy in patients with inflammatory bowel disease. Gut 2011;60:A219.

12 Mohammed N, Kant P, Abid F, et al. OC-028 High definition white light endoscopy (HDWLE) versus high definition with chromoendoscopy (HDCE) in the detection of dysplasia in long standing ulcerative colitis: a randomised controlled trial. Gut 2015:64:A14:2-15.

13 lacucci M, Kaplan GG, Panaccione $R$, et al. A randomized trial comparing high definition colonoscopy alone with high definition dye spraying and electronic virtual Chromoendoscopy for detection of colonic neoplastic lesions during IBD surveillance colonoscopy. Am J Gastroentero/ 2018;113:225-34.

14 lannone A, Ruospo M, Palmer SC, et al. Systematic review with network metaanalysis: endoscopic techniques for dysplasia surveillance in inflammatory bowel disease. Aliment Pharmacol Ther 2019:50:858-71.

15 Bisschops R, Bessissow T, Joseph JA, et al. Chromoendoscopy versus narrow band imaging in UC: a prospective randomised controlled trial. Gut 2018;67:1087-94.

16 Veitch AM, Vanbiervliet G, Gershlick AH. Endoscopy in patients on antiplatelet or anticoagulant therapy, including direct oral anticoagulants. In: British Society of gastroenterology (Bsg) and European Society of gastrointestinal endoscopy (ESGE) guidelines, 2016

17 Dekker E, van den Broek FJ, Reitsma JB, et al. Narrow-Band imaging compared with conventional colonoscopy for the detection of dysplasia in patients with longstanding ulcerative colitis. Endoscopy 2007;39:216-21.

18 Pohl J, Lotterer E, Balzer C, et al. Computed virtual chromoendoscopy versus standard colonoscopy with targeted indigocarmine chromoscopy: a randomised multicentre trial. Gut 2009:58:73-8

19 Pellisé M, López-Cerón M, Rodríguez de Miguel C, et al. Narrow-Band imaging as an alternative to chromoendoscopy for the detection of dysplasia in long-standing inflammatory bowel disease: a prospective, randomized, crossover study. Gastrointest Endosc 2011;74:840-8

20 lacucci M, Kaplan GG, Panaccione $\mathrm{R}$, et al. A randomized trial comparing high definition colonoscopy alone with high definition dye spraying and electronic virtual Chromoendoscopy for detection of colonic neoplastic lesions during IBD surveillance colonoscopy. Am J Gastroentero/ 2018;113:225-34.

21 Laine L, Kaltenbach T, Barkun A, et al. Scenic international consensus statement on surveillance and management of dysplasia in inflammatory bowel disease. Gastroenterology 2015;148:e28:639-51.

22 Picco MF, Pasha S, Leighton JA, et al. Procedure time and the determination of polypoid abnormalities with experience: implementation of a chromoendoscopy program for surveillance colonoscopy for ulcerative colitis. Inflamm Bowel Dis 2013;19:1913-20.

23 Bisschops R, East JE, Hassan C, et al. Advanced imaging for detection and differentiation of colorectal neoplasia: European Society of Gastrointestinal Endoscopy (ESGE) Guideline - Update 2019. Endoscopy 2019;51:1155-79.

24 Ignjatovic A, East JE, Subramanian V, et al. Narrow band imaging for detection of dysplasia in colitis: a randomized controlled trial. Am J Gastroenterol 2012;107:885-90.

25 Gallinger ZR, Rumman A, Murthy SK, et al. Perspectives on endoscopic surveillance of dysplasia in inflammatory bowel disease: a survey of academic Gastroenterologists. Endosc Int Open 2017;5:E974-9.

26 Vienne A, Simon T, Cosnes J, et al. Low prevalence of colonoscopic surveillance of inflammatory bowel disease patients with longstanding extensive colitis: a clinical practice survey nested in the CESAME cohort. Aliment Pharmacol Ther 2011:34:188-95.

27 Watanabe T, Ajioka Y, Mitsuyama K, et al. Comparison of Targeted vs Random Biopsies for Surveillance of Ulcerative Colitis-Associated Colorectal Cancer. Gastroenterology 2016;151:1122-30

28 Gasia MF, Ghosh S, Panaccione R, et al. Targeted biopsies identify larger proportions of patients with colonic neoplasia undergoing high-definition colonoscopy, dye Chromoendoscopy, or electronic virtual Chromoendoscopy. Clin Gastroenterol Hepatol 2016;14:704-12 
29 Jung Y, Joo Y-E, Kim HG, et al. Relationship between the endoscopic withdrawal time and adenoma/polyp detection rate in individual colonic segments: a KASID multicenter study. Gastrointest Endosc 2019;89:523-30.
30 lacucci M, Cannatelli R, Tontini GE, et al. Improving the quality of surveillance colonoscopy in inflammatory bowel disease. Lancet Gastroenterol Hepatol 2019;4:971-83. 